\title{
X-Ray Filter Thickness Minimum
}

National Cancer Institute

\section{Source}

National Cancer Institute. X-Ray Filter Thickness Minimum. NCI Thesaurus. Code C69241.

The minimum thickness of the X-ray absorbing material used in the filter. 